Bull. Austral. Math. Soc.

$06 \mathrm{C} 15,03 \mathrm{G} 12$

VOL. 64 (2001) [81-98]

\title{
BLOCKS OF HOMOGENEOUS EFFECT ALGEBRAS
}

\author{
GejZA Jenča
}

\begin{abstract}
Effect algebras, introduced by Foulis and Bennett in 1994, are partial algebras which generalise some well known classes of algebraic structures (for example orthomodular lattices, MV algebras, orthoalgebras et cetera). In the present paper, we introduce a new class of effect algebras, called homogeneous effect algebras. This class includes orthoalgebras, lattice ordered effect algebras and effect algebras satisfying the Riesz decomposition property. We prove that every homogeneous effect algebra is a union of its blocks, which we define as maximal sub-effect algebras satisfying the Riesz decomposition property. This generalises a recent result by Riečanová, in which lattice ordered effect algebras were considered. Moreover, the notion of a block of a homogeneous effect algebra is a generalisation of the notion of a block of an orthoalgebra. We prove that the set of all sharp elements in a homogeneous effect algebra $E$ forms an orthoalgebra $E_{S}$. Every block of $E_{S}$ is the centre of a block of $E$. The set of all sharp elements in the compatibility centre of $E$ coincides with the centre of $E$. Finally, we present some examples of homogeneous effect algebras and we prove that for a Hilbert space $\mathbb{H}$ with $\operatorname{dim}(\mathbb{H})>1$, the standard effect algebra $\mathcal{E}(\mathbb{H})$ of all effects in $\mathbb{H}$ is not homogeneous.
\end{abstract}

\section{INTRODUCTION}

Effect algebras (or D-posets) have recently been introduced by Foulis and Bennett in [8] for the study of foundations of quantum mechanics. (See also [15, 10].) The prototype effect algebra is $(\mathcal{E}(\mathbb{H}), \oplus, 0, I)$, where $\mathbb{H}$ is a Hilbert space and $\mathcal{E}(\mathbb{H})$ consists of all self-adjoint operators $A$ of $\mathbb{H}$ such that $0 \leqslant A \leqslant I$. For $A, B \in \mathcal{E}(\mathbb{H}), A \oplus B$ is defined if and only if $A+B \leqslant 1$ and then $A \oplus B=A+B$. $\mathcal{E}$ (III) plays an important role in the foundations of quantum mechanics $[16,3]$.

The class of effect algebras includes orthoalgebras [9] and a subclass (called MV-effect algebras or Boolean. D-posets or Boolean effect algebras), which is essentially equivalent to MV-algebras, introduced by Chang in [4] (see for example, [6, 1] for results on MValgebras in the context of effect algebras). The class of orthoalgebras includes other classes of well-known sharp structures, like orthomodular posets [17] and orthomodular lattices $[14,2]$.

Received 2nd October, 2000

This research is supported by grant G-1/7625/20 of MS̃ SR, Slovakia

Copyright Clearance Centre, Inc. Serial-fee code: 0004-9727/01 \$A2.00+0.00. 
One of the most important results in the theory of effect algebras was proved by Riečanová in her paper [20]. She proved that every lattice ordered effect algebra is a union of maximal mutually compatible sub-effect algebras, called blocks. This result generalises the well-known fact that an orthomodular lattice is a union of its maximal Boolean subalgebras. Moreover, as proved in [13], in every lattice ordered effect algebra $E$ the set of all sharp elements forms a sub-effect algebra $E_{S}$, which is a sub-lattice of $E$; $E_{S}$ is then an orthomodular lattice, and every block of $E_{S}$ is the centre of some block of $E$. On the other hand, every orthoalgebra is a union of maximal Boolean sub-orthoalgebras. Thus, although the classes of lattice ordered effect algebras and orthoalgebras are independent, both-lattice ordered effect algebras and orthoalegebras are covered by their blocks. This observation leads us to a natural question:

QUESTION 1.1. Is there a class of effect algebras, say $\mathbf{X}$, with the following properties?

- $\mathbf{X}$ includes orthoalgebras and lattice ordered effect algebras.

- Every $E \in \mathbf{X}$ is a union of (some sort of) blocks.

In the present paper, we answer this question in the affirmative. We introduce a new class of effect algebras, called homogeneous effect algebras. This class includes lattice ordered effect algebras, orthoalgebras and effect algebras satisfying the Riesz decomposition property (see for example [18]). The blocks in homogeneous algebras are maximal sub-effect algebras satisfying the Riesz decomposition property. We prove that the set of all sharp elements $E_{S}$ in a homogeneous effect algebra $E$ forms a sub-effect algebra (of course, $E_{S}$ is an orthoalgebra) and every block of $E_{S}$ is the centre of a block of $E$. In the last section we present some examples of homogeneous effect algebras and we prove that $\mathcal{E}(\mathbb{H})$ is not homogeneous unless $\operatorname{dim}(\mathbb{H}) \leqslant 1$.

\section{DEFINITIONS AND BASIC RELATIONSHIPS}

An effect algebra is a partial algebra $(E ; \oplus, 0,1)$ with a binary partial operation $\oplus$ and two nullary operations 0,1 satisfying the following conditions.

(E1) If $a \oplus b$ is defined, then $b \oplus a$ is defined and $a \oplus b=b \oplus a$.

(E2) If $a \oplus b$ and $(a \oplus b) \oplus c$ are defined, then $b \oplus c$ and $a \oplus(b \oplus c)$ are defined and $(a \oplus b) \oplus c=a \oplus(b \oplus c)$.

(E3) For every $a \in E$ there is a unique $a^{\prime} \in E$ such that $a \oplus a^{\prime}=1$.

(E4) If $a \oplus 1$ exists, then $a=0$

Effect algebras were introduced by Foulis and Bennett in their paper [8]. Independently, Kôpka and Chovanec introduced an essentially equivalent structure called a $D$-poset (see [15]). Another equivalent structure, called a weak orthoalgebra was introduced by Giuntini and Greuling in [10].

For brevity, we denote the effect algebra $(E, \oplus, 0,1)$ by $E$. In an effect algebra $E$, we write $a \leqslant b$ if and only if there is $c \in E$ such that $a \oplus c=b$. It is easy to check that 
every effect algebra is cancellative, thus $\leqslant$ is a partial order on $E$. In this partial order, 0 is the least and 1 is the greatest element of $E$. Moreover, it is possible to introduce a new partial operation $\ominus ; b \ominus a$ is defined if and only if $a \leqslant b$ and then $a \oplus(b \ominus a)=b$. It can be proved that $a \oplus b$ is defined if and only if $a \leqslant b^{\prime}$ if and only if $b \leqslant a^{\prime}$. Therefore, it is usual to denote the domain of $\oplus$ by $\perp$. If $a \perp b$, we say that $a$ and $b$ are orthogonal. Let $E_{0} \subseteq E$ be such that $1 \in E_{0}$ and, for all $a, b \in E_{0}$ with $a \geqslant b, a \Theta b \in E_{0}$. Since $a^{\prime}=1 \ominus a$ and $a \oplus b=\left(a^{\prime} \ominus b\right)^{\prime}, E_{0}$ is closed with respect to $\oplus$ and '. We then say that $\left(E_{0}, \oplus, 0,1\right)$ is a sub-effect algebra of $E$. Another possibility to construct a substructure of an effect algebra $E$ is to restrict $\oplus$ to an interval $[0, a]$, where $a \in E$, letting $a$ act as the unit element. We denote such an effect algebra by $[0, a]_{E}$.

Remark. For our purposes, it is natural to consider orthomodular lattices, orthomodular posets, MV-algebras, and Boolean algebras as special types of effect algebras. In the present paper, we shall write briefly "orthomodular lattice" instead of "effect algebra associated with an orthomodular lattice" and similarly for orthomodular posets, MValgebras, and Boolean algebras.

An effect algebra satisfying $a \perp a \Longrightarrow a=0$ is called an orthoalgebra (see [9]). An effect algebra $E$ is an orthomodular poset if and only if, for all $a, b, c \in E, a \perp b \perp c \perp a$ implies that $a \oplus b \oplus c$ exists (see [8]). An orthoalgebra is an orthomodular lattice if and only if it is lattice ordered.

Let $E$ be an effect algebra. Let $C=\left(c_{1}, \ldots, c_{n}\right)$ be a finite family of elements of $E$. We say that $C$ is orthogonal if and only if the sum $c_{1} \oplus \cdots \oplus c_{n}$ exists. We then write $\bigoplus C=c_{1} \oplus \cdots \oplus c_{n}$. For $n=0$, we put $\oplus C=0$. We say that $\operatorname{Ran}(C)=\left\{c_{1}, \ldots, c_{n}\right\}$ is the range of $C$. Let $C=\left(c_{1}, \ldots, c_{n}\right), D=\left(d_{1}, \ldots, d_{k}\right)$ be orthogonal families of elements. We say that $D$ is a refinement of $C$ if and only if there is a partition $P=\left\{P_{1}, \ldots, P_{n}\right\}$ of $\{1, \ldots, k\}$ such that, for all $1 \leqslant i \leqslant n, c_{i}=\bigoplus_{j \in P_{i}} d_{j}$. Note that if $D$ is a refinement of $C$, then $\oplus C=\oplus D$.

A finite subset $M_{F}$ of an effect algebra $E$ is called compatible with cover in $X \subseteq E$ if and only if there is a finite orthogonal family $C=\left(c_{1}, \ldots, c_{n}\right)$ with $\operatorname{Ran}(C) \subseteq X$ such that for every $a \in M_{F}$ there is a set $A \subseteq\{1, \ldots, n\}$ with $a=\bigoplus_{i \in A} c_{i} . C$ is then called an orthogonal cover of $M_{F}$. A subset $M$ of $E$ is called compatible with covers in $X \subseteq E$ if and only if every finite subset of $M$ is compatible with cover in $X$. A subset $M$ of $E$ is called internally compatible if and only if $M$ is compatible with covers in $M$. A subset $M$ of $E$ is called compatible if and only if $M$ is compatible with covers in $E$. An effect algebra $E$ is said to be compatible if $E$ is a compatible subset of $E$. If $\{a, b\}$ is a compatible set, we write $a \leftrightarrow b$. It is easy to check that $a \leftrightarrow b$ if and only if there are $a_{1}, b_{1}, c \in E$ such that $a_{1} \oplus c=a, b_{1} \oplus c=b$, and $a_{1} \oplus b_{1} \oplus c$ exists. A subset $M$ of $E$ is called mutually compatible if and only if, for all $a, b \in M, a \leftrightarrow b$. Obviously, every compatible subset of an effect algebra is mutually compatible. In the class of lattice 
ordered effect algebras, the converse also holds. It is well known that in an orthomodular poset, a mutually compatible set need not to be compatible (see for example [17]).

A lattice ordered effect algebra $E$ is called an $M V$-algebra if and only if $E$ is compatible (see [6]). An MV-algebra which is an orthoalgebra is a Boolean algebra. Recently, Z. Riečanová proved in her paper [20] that every lattice ordered effect algebra is a union of MV-algebras, which are maximal mutually compatible subsets. These are called blocks. She proved that every block of a lattice ordered effect algebra $E$ is a sub-effect algebra and a sublattice of $E$. Note that Riečanová's results imply that every mutually compatible subset of a lattice ordered effect algebra is compatible. Indeed, let $M$ be a mutually compatible set. Then $M$ can be embedded into a block $B$, which is an MV-algebra and hence compatible. Since $B$ is compatible and $M \subseteq B, M$ is compatible.

On the other hand, it is easy to prove that every element of an orthoalgebra can be embedded into a maximal sub-orthoalgebra, which is a Boolean algebra.

We say that an effect algebra $E$ satisfies the Riesz decomposition property if and only if, for all $u, v_{1}, \ldots, v_{n} \in E$ such that $v_{1} \oplus \cdots \oplus v_{n}$ exists and $u \leqslant v_{1} \oplus \cdots \oplus v_{n}$, there are $u_{1}, \ldots, u_{n} \in E$ such that, for all $1 \leqslant i \leqslant n, u_{i} \leqslant v_{i}$ and $u=u_{1} \oplus \cdots \oplus u_{n}$. It is easy to check that an effect algebra $E$ satisfies the Riesz decomposition property if and only if $E$ satisfies the Riesz decomposition property with fixed $n=2$. A lattice ordered effect algebra $E$ satisfies the Riesz decomposition property if and only if $E$ is an MV-algebra. An orthoalgebra $E$ satisfies the Riesz decomposition property if and only if $E$ is a Boolean algebra.

Let $E_{1}, E_{2}$ be effect algebras. A map $\phi: E_{1} \mapsto E_{2}$ is called a morphism if and only if $\phi(1)=1$ and $a \perp b$ implies that $\phi(a) \perp \phi(b)$ and then $\phi(a \oplus b)=\phi(a) \oplus \phi(b)$. A morphism $\phi$ is an isomorphism if and only if $\phi$ is bijective and $\phi^{-1}$ is a morphism.

DEFinition 2.1: An effect algebra $E$ is called homogeneous if and only if, for all $u, v_{1}, v_{2} \in E$ such that $v_{1} \perp v_{2}, u \leqslant v_{1} \oplus v_{2}, u \leqslant\left(v_{1} \oplus v_{2}\right)^{\prime}$, there are $u_{1}, u_{2}$ such that $u_{1} \leqslant v_{1}, u_{2} \leqslant v_{2}$ and $u=u_{1} \oplus u_{2}$.

\section{Proposition 2.2.}

(a) Every orthoalgebra is homogeneous.

(b) Every effect algebra satisfying the Riesz decomposition property is homogeneous.

(c) Every lattice ordered effect algebra is homogeneous.

ProOF: For the proof of (a), observe that $u \leqslant v_{1} \oplus v_{2}$ and $u \leqslant\left(v_{1} \oplus v_{2}\right)^{\prime}$ imply that $u \perp u$ and thus $u=0$. (b) is obvious. For the proof of (c), let $E$ be a lattice ordered effect algebra. Note that $v_{1} \perp v_{2}, u \leqslant\left(v_{1} \oplus v_{2}\right)^{\prime}$ imply that the set $\left\{u, v_{1}, v_{2}\right\}$ is mutually orthogonal and thus mutually compatible. Therefore, by [20], $\left\{u, v_{1}, v_{2}\right\}$ can be embedded into a block $B$. Since $B$ is an MV-algebra, $B$ satisfies the Riesz decomposition property, hence $E$ is homogeneous. 
Proposition 2.3. Let $E$ be a homogeneous effect algebra. Let $u, v_{1}, \ldots, v_{n} \in$ $E$ be such that $v_{1} \oplus \cdots \oplus v_{n}$ exists, $u \leqslant v_{1} \oplus \cdots \oplus v_{n}$ and $u \leqslant\left(v_{1} \oplus \cdots \oplus v_{n}\right)^{\prime}$. Then there are $v_{1}, \ldots, v_{n}$ such that, for all $1 \leqslant i \leqslant n, v_{i} \leqslant u_{i}$ and $u=u_{1} \oplus \cdots \oplus u_{n}$.

Proof: (By induction.) For $n=1$, it suffices to put $u_{1}=u$. Assume that the proposition holds for $n=k$. Let $u, v_{1}, \ldots, v_{k+1}$ be such that $v_{1} \oplus \cdots \oplus v_{k+1}$ exists, $u \leqslant v_{1} \oplus v_{2} \oplus \cdots \oplus v_{k+1}$ and $u \leqslant\left(v_{1} \oplus v_{2} \oplus \cdots \oplus v_{k+1}\right)^{\prime}$. Since $E$ is homogeneous, there are $u_{1} \leqslant v_{1}$ and $z \leqslant v_{2} \oplus \cdots \oplus v_{k+1}$ such that $u=u_{1} \oplus z$. Since

$$
z \leqslant u \leqslant\left(v_{1} \oplus \cdots \oplus v_{k+1}\right)^{\prime} \leqslant\left(v_{2} \oplus \cdots \oplus v_{k+1}\right)^{\prime},
$$

we see that $z \leqslant\left(v_{2} \oplus \cdots \oplus v_{k+1}\right)^{\prime}$. Thus, we may apply induction hypothesis. The rest is trivial.

\section{BLOCKS OF HOMOGENEOUS EFFECT ALGEBRAS}

Let $E$ be an effect algebra. We say that a sub-effect algebra $B$ of $E$ is a block of $E$ if and only if $B$ is a maximal sub-effect algebra satisfying the Riesz decomposition property. This definition of a block is consistent with the definition of a block of the theory of orthoalgebras (maximal Boolean sub-orthoalgebra) and also in the theory of lattice ordered effect algebras (maximal mutually compatible subset).

In this section, we prove that blocks of homogeneous effect algebras coincide with the maximal internally compatible subsets, which contain 1. As a consequence, every homogeneous effect algebra is a union of its blocks.

The main tool we use is the closure operation $M \mapsto \bar{M}$ which is defined on the system of all subsets of an effect algebra $E$ in the following way. Let $M$ be a subset of an effect algebra $E$. First we define certain subsets $M_{n}(n \in \mathbb{N})$ of $E$ as follows : $M_{0}=M$ and for $n \in \mathbb{N}$

$$
M_{n+1}=\left\{x: x \leqslant y, y^{\prime} \text { for some } y \in M_{n}\right\} \cup\left\{y \ominus x: x \leqslant y, y^{\prime} \text { for some } y \in M_{n}\right\} .
$$

Then we put $\bar{M}=\bigcup_{n \in \mathbb{N}} M_{n}$. Note that, for all $n \in \mathbb{N}, M_{n} \subseteq M_{n+1}$ and that $\overline{\bar{M}}=\bar{M}$. In an orthoalgebra, $M=\bar{M} \cup\{0\}$ for every set $M$.

LEMMA 3.1. Let $E$ be an effect algebra. Let $M$ be an compatible subset of $E$. Then $M$ can be embedded into a maximal compatible subset of $E$.

Proof: The proof is an easy application of Zorn's lemma and is left to the reader.

Proposition 3.2. Let $E$ be a homogeneous effect algebra. Let $M \subseteq E$ be a finite compatible set, $a, b \in M, a \geqslant b$. Let $C=\left(c_{1}, \ldots, c_{k}\right)$ be an orthogonal cover of $M$. Let $A, B \subseteq\{1, \ldots, k\}$ be such that $a=\bigoplus_{i \in A} c_{i}$ and $b=\bigoplus_{i \in B} c_{i}$. Then, there is a refinement of $C$, say $W=\left(w_{1}, \ldots, w_{n}\right)$ and sets $B_{W} \subseteq A_{W} \subseteq\{1, \ldots, n\}$ such that $\left(w_{i}\right)_{i \in A_{W}}$ is a refinement of $\left(c_{i}\right)_{i \in A}$ and $\left(w_{i}\right)_{i \in B_{W}}$ is a refinement of $\left(c_{i}\right)_{i \in B}$. Moreover, we have $\operatorname{Ran}(W) \subseteq \overline{\operatorname{Ran}(C)}$. 
Proof: If $|B \backslash A|=0$ then $B \subseteq A$ and there is nothing to prove.

Let $l \in \mathbb{N}$. Assume that Proposition 3.2 holds for all $C, A, B$ with $|B \backslash A|=l$. Let $C_{0}, A_{0}, B_{0}$ be as in the assumption of Proposition 3.2, with $\left|B_{0} \backslash A_{0}\right|=l+1$.

To avoid double indices, we may safely assume that $A_{0}$ and $B_{0}$ are such that, for some $0 \leqslant r, s, t \leqslant k, B_{0} \backslash A_{0}=\{1, \ldots, r\}, B_{0} \cap A_{0}=\{r+1, \ldots, s\}, A_{0} \backslash B_{0}=\{s+1, \ldots, t\}$.

Write $b_{1}=c_{1} \oplus \cdots \oplus c_{l+1}, d=c_{l+2} \oplus: \cdots \oplus c_{s}, a_{1}=c_{s+1} \oplus \cdots \oplus c_{t}$. Since $b_{1} \oplus d=b \leqslant$ $a=a_{1} \oplus d$, we see that $c_{l+1} \leqslant b_{1} \leqslant a_{1}$. Since $C_{0}$ is an orthogonal family, $c_{l+1} \leqslant a_{1}{ }^{\prime}$. By Proposition 2.3, this implies that there are $v_{s+1}, \ldots, v_{t}$ such that, for all $s+1 \leqslant i \leqslant t$, $v_{i} \leqslant c_{i}$ and $c_{l+1}=v_{s+1} \oplus \cdots \oplus v_{t}$. Let us construct a refinement of $C_{0}$, say $C_{1}=\left(e_{i}\right)$, as follows.

$$
C_{1}=\left(c_{1}, \ldots, c_{l}, v_{s+1}, \ldots, v_{t}, c_{l+2}, \ldots, c_{s}, c_{s+1} \ominus v_{s+1}, \ldots, c_{t} \ominus v_{t}, c_{t+1}, \ldots, c_{k}, c_{l+1}\right)
$$

Obviously, $C_{1}$ is a refinement of $C_{0}$ and $\operatorname{Ran}\left(C_{1}\right) \subseteq \overline{\operatorname{Ran}\left(C_{0}\right)}$. Moreover, we have

$$
b=\bigoplus\left(c_{1}, \ldots, c_{l}, v_{s+1}, \ldots, v_{t}, c_{l+2}, \ldots, c_{s}\right)
$$

and

$$
a=\bigoplus\left(v_{s+1}, \ldots, v_{t}, c_{l+2}, \ldots, c_{s}, c_{s+1} \ominus v_{s+1}, \ldots, c_{t} \ominus v_{t}\right)
$$

By the latter equations, we can find sets $A_{1}, B_{1}$ of indices such that $a=\bigoplus_{i \in A_{1}} e_{i}, b=\bigoplus_{i \in B_{1}} e_{i}$ and $B_{1} \backslash A_{1}=\{1, \ldots, l\}$. Moreover, $\left(e_{i}\right)_{i \in A_{1}}$ is a refinement of $\left(c_{i}\right)_{i \in A_{0}}$ and $\left(e_{i}\right)_{i \in B_{1}}$ is a refinement of $\left(c_{i}\right)_{i \in B_{0}}$. As $\left|B_{1} \backslash A_{1}\right|=l$, we may apply the induction hypothesis on $C_{1}, A_{1}, B_{1}$ to find a refinement $W=\left(w_{1}, \ldots, w_{n}\right)$ of $C_{1}$ with $\operatorname{Ran}(W) \subseteq \overline{\operatorname{Ran}\left(C_{1}\right)}$ and sets $B_{W} \subseteq A_{W} \subseteq\{1, \ldots, n\}$ such that $\left(w_{i}\right)_{i \in A_{W}}$ is a refinement of $\left(e_{i}\right)_{i \in A_{1}}$ and $\left(w_{i}\right)_{i \in B_{W}}$ is a refinement of $\left(e_{i}\right)_{i \in B_{1}}$. Obviously, $W$ is a refinement of $C_{0}$ and we see that

$$
\operatorname{Ran}(W) \subseteq \overline{\operatorname{Ran}\left(C_{1}\right)} \subseteq \overline{\overline{\operatorname{Ran}\left(C_{0}\right)}}=\overline{\operatorname{Ran}\left(C_{0}\right)}
$$

Similarly, $\left(w_{i}\right)_{i \in A_{W}}$ is a refinement of $\left(c_{i}\right)_{i \in A_{0}}$ and $\left(w_{i}\right)_{i \in B_{W}}$ is a refinement of $\left(c_{i}\right)_{i \in B_{0}}$. This concludes the proof.

Corollary 3.3. Let $M$ be a finite compatible subset of a homogeneous effect algebra $E$. Let $a, b \in M$ be such that $a \geqslant b$. Then $M \cup\{a \ominus b\}$ is a compatible set. Proof: Let $W, A_{W}, B_{W}$ be as in Proposition 3.2. Then $a \ominus b=\bigoplus_{i \in A_{W} \backslash B_{W}} w_{i}$, so $W$
is an orthogonal cover of $M \cup\{a \ominus b\}$.

Corollary 3.4. Let $M$ be a finite compatible subset of a homogeneous effect algebra $E$. Let $a, b \in M$ be such that $a \perp b$. Then $M \cup\{a \oplus b\}$ is a compatible set.

PRoof: It is easy to check that, for every compatible set $M_{0}, M_{0} \cup M_{0}{ }^{\prime}=M_{0} \cup\left\{a^{\prime}\right.$ : $\left.-a \in M_{0}\right\}$ is a compatible set. The rest follows from Corollary 3.3 and from the equation $a \oplus b=\left(a^{\prime} \ominus b\right)^{\prime}$.

THEOREM 3.5. Let $E$ be an effect algebra. The following are equivalent. 
(a) E satisfies the Riesz decomposition property.

(b) $E$ is homogeneous and compatible.

Proof: (a) implies (b): It is evident that $E$ is homogeneous. It remains to prove that every $n$-element subset of $E$ is compatible. For $n=1$, there is nothing to prove. For $n>1$, let us assume that every $(n-1)$-element subset of $E$ is compatible. Let $X=\left\{x_{1}, \ldots, x_{n}\right\}$ be a subset of $E$. By the induction hypothesis, $X_{0}=\left\{x_{1}, \ldots, x_{n-1}\right\}$ is compatible. Thus, there is an orthogonal cover of $X_{0}$, say $C=\left(c_{1}, \ldots, c_{k}\right)$. Since $x_{n} \leqslant(\bigoplus C) \oplus(\bigoplus C)^{\prime}$ and $E$ satisfies the Riesz decomposition property, there exist $y_{1}, y_{2}$ such that $y_{1} \leqslant(\bigoplus C), y_{2} \leqslant(\bigoplus C)^{\prime}$ and $x_{n}=y_{1} \oplus y_{2}$. Since $y_{1} \leqslant(\bigoplus C)$, there are $z_{1}, \ldots, z_{k}$ such that, for all $1 \leqslant i \leqslant k, z_{i} \leqslant c_{i}$ and $y_{1}=z_{1} \oplus \cdots \oplus z_{k}$. Consequently,

$$
\left(z_{1}, c_{1} \ominus z_{1}, \ldots, z_{k}, c_{k} \ominus z_{k}, y_{2}\right)
$$

is an orthogonal cover of $X$ and $X$ is compatible.

(b) implies (a): Let $u, v_{1}, v_{2} \in E$ be such that $v_{1} \perp v_{2}, u \leqslant v_{1} \oplus v_{2}$. If $v_{1}=0$ or $v_{2}=0$, there is nothing to prove. Thus, let us assume that $v_{1}, v_{2} \neq 0$. By Proposition 3.2, $v_{1} \leqslant v_{1} \oplus v_{2}$ implies that there an orthogonal cover $W=\left(w_{1}, \ldots, w_{m}\right)$ of $\left\{u, v_{1}, v_{2}, v_{1} \oplus v_{2}\right\}$ such that, for some $V_{1} \subseteq V \subseteq\{1, \ldots, m\}$, we have $\bigoplus_{i \in V} w_{i}=v_{1} \oplus v_{2}$ and $\bigoplus_{i \in V_{1}} w_{i}=v_{1}$. This implies that $\bigoplus_{i \in V \backslash V_{1}} w_{i}=v_{2}$. By Proposition 3.2, $u \leqslant v_{1} \oplus v_{2}$ implies that there is a refinement of $W$, say $Q=\left(q_{1}, \ldots, q_{n}\right)$, and some $U \subseteq Z \subseteq\{1, \ldots, n\}$ such that $\bigoplus_{i \in U} q_{i}=u$ and $\bigoplus_{i \in Z} q_{i}=v_{1} \oplus v_{2}$. Moreover, by Proposition 3.2, we may assume that $\left(q_{i}\right)_{i \in Z}$ is a refinement of $\left(w_{i}\right)_{i \in V}$. This implies that there is $Z_{1} \subseteq Z$ such that $\bigoplus_{i \in Z_{1}} q_{i}=v_{1}$. Put $u_{1}=\bigoplus_{i \in U \cap Z_{1}} q_{i}$ and $u_{2}=\bigoplus_{i \in U \cap\left(Z \backslash Z_{1}\right)} q_{i}$. It remains to observe that $u=u_{1} \oplus u_{2}, u_{1} \leqslant v_{1}$ and $u_{2} \leqslant v_{2}$.

EXAMPLE 3.6. Let $R_{6}$ be a 6-element effect algebra with two atoms $\{a, b\}$, satisfying the equation $a \oplus a \oplus a=a \oplus b \oplus b=1$. Since $(a, b, b)$ is an orthogonal cover of $R_{6}$, $R_{6}$ is a compatible effect algebra. However, $R_{6}$ does not satisfy the Riesz decomposition property, since $a \leqslant b \oplus b$ and $a \wedge b=0$. This example shows that there are compatible effect algebras that do not satisfy the Riesz decomposition property.

Proposit ION 3.7. Let $M$ be a subset of a homogeneous effect algebra $E$ such that $M$ is compatible with covers in $\bar{M}$. Then $\bar{M}$ is internally compatible.

Proof: Consider (1). Since each finite subset of $\bar{M}$ can be embedded into some $M_{n}$, it suffices to prove that, for all $n \in \mathbb{N}, M_{n}$ is compatible with covers in $\bar{M}$. By assumption, $M=M_{0}$ is compatible with covers in $\bar{M}$. Assume that, for some $n \in \mathbb{N}, M_{n}$ is compatible with covers in $\bar{M}$. Obviously, every finite subset of $M_{n+1}$ can be embedded into a set of the form

$$
\left\{x_{1}, y_{1} \ominus x_{1}, \ldots, x_{k}, y_{k} \ominus x_{k}\right\} \subseteq M_{n+1}
$$


where for all $1 \leqslant i \leqslant k$ we have $x_{i} \leqslant y_{i}, y_{i}^{\prime}$ and $y_{i} \in M_{n}$.

We now prove the following

ClaIM. Let $x_{i}, y_{i}$ be as above. For every cover $C_{0}$ of $\left\{y_{1}, \ldots, y_{k}\right\}$, there is a refinement $W$ of $C_{0}$ such that $W$ covers $\left\{x_{1}, y_{1} \ominus x_{1}, \ldots, x_{k}, y_{k} \ominus x_{k}\right\}$ and $\operatorname{Ran}(W) \subseteq \overline{\operatorname{Ran}\left(C_{0}\right)}$.

Proof of the Claim: For $k=0$, we may put $W=C_{0}$. Assume that the Claim is satisfied for some $k=l \in \mathbb{N}$. Let $C_{0}$ be a cover of $\left\{y_{1}, \ldots, y_{l+1}\right\} \subseteq M_{n}$. Since $C_{0}$ is a cover of $\left\{y_{1}, \ldots, y_{l}\right\}$ as well, by the induction hypothesis there is a refinement of $C_{0}$, say $C_{1}$, such that $C_{1}$ covers $\left\{x_{1}, y_{1} \ominus x_{1}, \ldots, x_{l}, y_{l} \ominus x_{l}\right\}$ and $\operatorname{Ran}\left(C_{1}\right) \subseteq \overline{\operatorname{Ran}\left(C_{0}\right)}$. As $C_{1}$ is a refinement of $C_{0}, C_{1}$ covers $\left\{y_{1}, \ldots, y_{l+1}\right\}$. Thus, there are $\left(c_{1}, \ldots, c_{m}\right) \subseteq C_{1}$ such that $y_{l+1}=c_{1} \oplus \cdots \oplus c_{m}$. Since $x_{l+1} \leqslant y_{l+1}, y_{l+1}$, Proposition 2.3 implies that there are $z_{1}, \ldots, z_{m}$ such that, for all $1 \leqslant i \leqslant m, z_{i} \leqslant c_{i}$ and $x_{l+1}=z_{1} \oplus \cdots \oplus z_{l}$. Let us construct a refinement $W$ of $C_{1}$ by replacing each of the $c_{i}$ 's by the pair $\left(z_{i}, c_{i} \ominus z_{i}\right)$. Then $W$ is a refinement of $C_{1}$ and $W$ covers $\left\{x_{1}, y_{1} \ominus x_{1}, \ldots, x_{l+1}, y_{l+1} \ominus x_{l+1}\right\}$. Moreover, for all $1 \leqslant i \leqslant m, z_{i} \leqslant x_{l+1} \leqslant y_{l+1}{ }^{\prime} \leqslant c_{i}^{\prime}$, hence

$$
\operatorname{Ran}(W) \subseteq \overline{\operatorname{Ran}\left(C_{1}\right)} \subseteq \overline{\overline{\operatorname{Ran}\left(C_{0}\right)}}=\overline{\operatorname{Ran}\left(C_{0}\right)} .
$$

Now, let $M_{F}$ be a finite subset of $M_{n+1}$. We may assume that $M_{F}$ is of the form (2). By the outer induction hypothesis, $M_{n}$ is compatible with covers in $\bar{M}$, thus $\left\{y_{1}, \ldots, y_{k}\right\}$ is compatible with cover in $\bar{M}$. Let $C$ be an orthogonal cover of $\left\{y_{1}, \ldots, y_{k}\right\}$ with $\operatorname{Ran}(C) \subseteq$ $\bar{M}$. By the Claim, there is a refinement $W$ of $C$, such that $W$ covers $M_{F}$ and $\operatorname{Ran}(W) \subseteq$ $\overline{\operatorname{Ran}(C)} \subseteq \overline{\bar{M}}=\bar{M}$. Thus, $M_{F}$ is compatible with covers in $\bar{M}$ and we see that $\bar{M}$ is internally compatible.

The following are immediate consequences of Proposition 3.7.

Corollary 3.8.

(a) Let $M$ be an internally compatible subset of a homogeneous effect algebra $E$. Then $\bar{M}$ is an internally compatible set.

(b) Let $M$ be a maximal internally compatible subset of a homogeneous effect algebra $E$. Then $M=\bar{M}$.

Proposition 3.9. Let $E$ be a homogeneous effect algebra, let $M$ be an internally compatible set with $M=\bar{M}$. Let $a, b \in M, a \geqslant b$. Then $M \cup\{a \ominus b\}$ is an internally compatible set.

Proof: Let $M_{F}$ be a finite subset of $M$. Since $M$ is internally compatible, there is an orthogonal cover $C$ of $M_{F} \cup\{a, b\}$ with $\operatorname{Ran}(C) \subseteq M$. By Corollary 3.3, $M_{F} \cup\{a, b, a \ominus b\}$ is then compatible with cover in $\overline{\operatorname{Ran}(C)}$. Therefore, $M_{F} \cup\{a \ominus b\}$ is compatible with cover in $\overline{\operatorname{Ran}(C)}$. Since $\overline{\operatorname{Ran}(C)} \subseteq \bar{M}=M, M \cup\{a \ominus b\}$ is an internally compatible set.

As we shall show later in Example 5.6, a sub-effect algebra of a homogeneous effect 
algebra need not to be homogeneous. However, we have the following relationship on the positive side.

Proposition 3.10. Let $E$ be a homogeneous effect algebra. Let $F$ be a subeffect algebra of $E$ such that $F=\bar{F}$, where the closure is taken in $E$. Then $F$ is homogeneous.

Proof: Let $u, v_{1}, v_{2} \in F$ be such that $u \leqslant v_{1} \oplus v_{2}$ and $u \leqslant\left(v_{1} \oplus v_{2}\right)^{\prime}$. Since $E$ is homogeneous, there are $u_{1}, u_{2} \in E$ such that $u_{1} \leqslant v_{1}, u_{2} \leqslant v_{2}$ and $u=u_{1} \oplus u_{2}$. For $i \in\{1,2\}$, we have $u_{i} \leqslant v_{1} \oplus v_{2}$ and $u_{i} \leqslant\left(v_{1} \oplus v_{2}\right)^{\prime}$. Thus, $u_{1}, u_{2} \in \bar{F}=F$ and $F$ is homogeneous.

ThEOREM 3.11. Let $E$ be a homogeneous effect algebra, let $B \subseteq E$. The following are equivalent.

(a) $B$ is a maximal internally compatible set with $1 \in B$.

(b) $B$ is a block.

Proof: Assume that (a) is satisfied. By Corollary 3.8, part (b), $B=\bar{B}$. By Proposition 3.9, this implies that for all $a, b \in B$ such that $a \geqslant b, B \cup\{a \ominus b\}$ is an internally compatible set. Therefore, by maximality of $B, B$ is closed with respect to $\theta$. Since $1 \in B, B$ is a sub-effect algebra of $E$. Since $B$ is an internally compatible set, $B$ is a compatible effect algebra. By Corollary 3.8(b), $B=\bar{B}$. By Proposition 3.10, this implies that $B$ is homogeneous. Since $B$ is homogeneous and compatible, Theorem 3.5 implies that $B$ satisfies the Riesz decomposition property.

Assume that (b) is satisfied. By Theorem 3.5, $B$ is an internally compatible subset. By Lemma 3.1, $B$ can be embedded into a maximal internally compatible subset $B_{\max }$ of $E$. By the above part of the proof, $1 \in B \subseteq B_{\max }$ implies that $B_{\max }$ is a block. Therefore, $B=B_{\max }$ and (a) is satisfied.

COROLlary 3.12 . Let $E$ be a homogeneous effect algebra. Every finite compatible subset of $E$ can be embedded into a block.

Proof: Let $M_{F}$ be a finite compatible subset of $E$. Let $C=\left(c_{1}, \ldots, c_{n}\right)$ be an orthogonal cover of $M_{F}$. Then $M_{F} \cup\{1\}$ is a compatible set, with cover $C^{+}=$ $\left(c_{1}, \ldots, c_{n},(\oplus C)^{\prime}\right)$. Thus, $M_{F} \cup\{1\} \cup \operatorname{Ran}\left(C^{+}\right)$is an internally compatible set containing 1. Therefore, by Lemma 3.1, $M_{F} \cup\{1\} \cup \operatorname{Ran}\left(C^{+}\right)$can be embedded into a maximal compatible subset $B$ with $1 \in B$. By Theorem $3.11, B$ is a block.

Corollary 3.13 . Let $E$ be a homogeneous effect algebra. Then

$$
E=\bigcup\{B: B \text { is a block of } E\} \text {. }
$$

Proof: By Corollary 3.12 .

COROLlaRY 3.14. For an effect algebra $E$, the following are equivalent. 
(a) $E$ is homogeneous.

(b) Every finite compatible subset can be embedded into a block.

(c) Every finite compatible subset can be embedded into a sub-effect algebra of $E$ satisfying the Riesz decomposition property.

(d) The range of every finite orthogonal family can be embedded into a block.

(e) The range of every finite orthogonal family can be embedded into a subeffect algebra satisfying the Riesz decomposition property.

(f) The range of every orthogonal family with three elements can be embedded into a block.

(g) The range of every orthogonal family with three elements can be embedded into a sub-effect algebra satisfying the Riesz decomposition property.

Proof: (a) $\Longrightarrow$ (b) is Corollary 3.12. The implication chains (b) $\Longrightarrow$ (c) $\Longrightarrow$ $(\mathrm{e}) \Longrightarrow(\mathrm{g})$ and $(\mathrm{b}) \Longrightarrow(\mathrm{d}) \Longrightarrow(\mathrm{f}) \Longrightarrow(\mathrm{g})$ are obvious. To prove that $(\mathrm{g}) \Longrightarrow(\mathrm{a})$, assume that $E$ is an effect algebra satisfying (g), and let $u, v_{1}, v_{2} \in E$ be such that $u \leqslant v_{1} \oplus v_{2}, u \leqslant\left(v_{1} \oplus v_{2}\right)^{\prime}$. Then $\left(u, v_{1}, v_{2}\right)$ is an orthogonal family with three elements. By $(\mathrm{g}),\left\{u, v_{1}, v_{2}\right\}$ can be embedded into a sub-effect algebra $R$ satisfying the Riesz decomposition property. Thus, there are $u_{1}, u_{2} \in R \subseteq E$ such that $u_{1} \leqslant v_{1}, u_{2} \leqslant v_{2}$ and $u=u_{1} \oplus u_{2}$. Hence, $E$ is homogeneous.

QUESTION 3.15. Can every compatible subset of a homogeneous effect algebra $E$ be embedded into a block? This is true for orthomodular posets (see for example [17]) and for lattice ordered effect algebras. By Theorem 3.11 and Lemma 3.1, this question reduces to the question, whether a compatible subset can be embedded into an internally compatible subset containing 1 .

\section{COMPATIBILITY CENTRE AND SHARP ELEMENTS}

For a homogeneous effect algebra $E$, we write

$$
K(E)=\bigcap\{B: B \text { is a block of } E\} .
$$

We say that $K(E)$ is the compatibility centre of $E$. Note that $K(E)=\overline{K(E)}$ and hence, by Proposition 3.10, $K(E)$ is homogeneous.

An element $a$ of an effect algebra is called sharp if and only if $a \wedge a^{\prime}=0$. We denote the set of all sharp elements of an effect algebra $E$ by $E_{S}$. It is obvious that an effect algebra $E$ is an orthoalgebra if and only if $E=E_{S}$. An element $a$ of an effect algebra $E$ is called principal if and only if the interval $[0, a]$ is closed with respect to $\oplus$. Clearly, every principal element in an effect algebra is sharp. A principal element $a$ of an effect algebra is called central if and only if for all $b \in E$ there is a unique decomposition $b=b_{1} \oplus b_{2}$ with $b_{1} \leqslant a, b_{2} \leqslant a^{\prime}$. The set of all central elements of an effect algebra $E$ is called the centre 
of $E$ and is denoted by $C(E)$. In [11], the centre of an effect algebra was introduced and the following properties of $C(E)$ were proved.

Proposition 4.1. Let $E$ be an effect algebra. Then

- $C(E)$ is a sub-effect algebra of $E$.

- $C(E)$ is a Boolean algebra. Moreover, for all $a \in C(E)$ and $x \in E, a \wedge x$ exists.

- For all $a \in C(E)$, the map $\phi: E \mapsto[0, a]_{E}$ given by $\phi(x)=a \wedge x$ is a morphism.

- For all $a \in C(E), E$ is naturally isomorphic to $[0, a]_{E} \times\left[0, a^{\prime}\right]_{E}$. Moreover, for all effect algebras $E_{1}, E_{2}$ such that there is an isomorphism $\phi: E \mapsto$ $E_{1} \times E_{2}, \phi^{-1}(1,0)$ and $\phi^{-1}(0,1)$ are central in $E$.

A subset $I$ of an effect algebra $E$ is called an ideal if and only if the following condition is satisfied : $a, b \in I, a \perp b$ is equivalent to $a \oplus b \in I$. An ideal $I$ is called a Riesz ideal if and only if, for all $i, a, b$ such that $i \in I, a \perp b$ and $i \leqslant a \oplus b$, there are $i_{1}, i_{2}$ such that $i_{1} \leqslant a, i_{2} \leqslant b$ and $i \leqslant i_{1} \oplus i_{2}$. Riesz ideals were introduced in [12].

For a lattice ordered effect algebra $E$, it was proved in [19], that $C(E)=K(E) \cap E_{S}$. Moreover, as proved in [13], for a lattice ordered effect algebra $E, E_{S}$ is a sublattice of $E$, a sub-effect algebra of $E$, and every block of $E_{S}$ is the centre of a block of $E$. In the remainder of this section, we shall extend some of these results to the class of homogeneous effect algebras.

PROPOSITION 4.2. Let $a$ be an element of a homogeneous effect algebra $E$. The following are equivalent.

(a) $a \in E_{S}$.

(b) $a$ is central in every block of $E$ which contains $a$.

(c) $a$ is central in some block of $E$.

Proof: (a) implies (b): Assume that $a \in E$ is sharp, let $B$ be a block of $E$ such that $a \in B$. Since $a$ is sharp in $E, a$ is sharp in $B$. We shall prove that $a$ is principal in $B$. Let $x_{1}, x_{2} \in B$ be such that $x_{1}, x_{2} \leqslant a, x_{1} \perp x_{2}$. Since $B$ is a sub-effect algebra of $E, x_{1} \oplus x_{2} \in B$. Since $B$ is internally compatible, $x_{1} \oplus x_{2} \leftrightarrow a$ in $B$. By [5], Lemma 2, $x_{1} \oplus x_{2} \leftrightarrow a$ in $B$ implies that there are $y_{1}, y_{2} \in B$ such that $y_{1} \leqslant a, y_{2} \leqslant a^{\prime}$ and $x_{1} \oplus x_{2}=y_{1} \oplus y_{2}$. Since $B$ satisfies the Riesz decomposition property, $y_{2} \leqslant x_{1} \oplus x_{2}$ implies that there are $t_{1}, t_{2} \in B$ such that $t_{1} \leqslant x_{1}, t_{2} \leqslant x_{2}$ and $y_{2}=t_{1} \oplus t_{2}$. For $i \in\{1,2\}$, $t_{i} \leqslant a, a^{\prime}$. Since $a$ is sharp in $B$, this implies that $t_{1}=t_{2}=0$. Thus, $x_{1} \oplus x_{2}=y_{1} \leqslant a$ and $a$ is principal in $B$ and hence $[0, a] \cap B$ is an ideal in $B$. Since $B$ satisfies the Riesz decomposition property, every ideal in $B$ is a Riesz ideal. By [5], an element $a$ of an effect algebra is central if and only if $[0, a]$ is a Riesz ideal. Therefore, $a$ is central in $B$.

(b) implies (c): By Corollary 3.13, every clement of $E$ is in some block. 
(c) implies (a): Let $a \in C(B)$ for some block $B$, let $b \leqslant a, a^{\prime}$. Since $B=\bar{B}, b \in B$. Thus, $b=0$ and $a$ is sharp.

COROLLARY 4.3. Let a be an element of an effect algebra $E$ satisfying the Riesz decomposition property. The following are equivalent.

(a) $a \in E_{S}$.

(b) $a \in C(E)$.

(c) $a$ is principal.

Proof: By Proposition 4.2, (a) is equivalent to (b). In every effect algebra, all principal elements are sharp. Every central element is principal.

Corollary 4.4. For a homogeneous effect algebra $E, E_{S}$ is a sub-effect algebra of $E$. Moreover, $E_{S}$ is an orthoalgebra.

Proof: Obviously, $0,1 \in E_{S}$ and $E_{S}$ is closed with respect to '. Assume $a, b \in E_{S}$, $a \perp b$. Then $\{a ; b\}$ is a finite compatible set. Thus, by Corollary $3.12,\{a, b\}$ can be embedded into a block $B$. By Proposition 4.2, $a, b \in C(B)$. Since $C(B)$ is a sub-effect algebra of $B, a \oplus b \in C(B)$. By Proposition 4.2, $C(B) \subseteq E_{S}$, thus $a \oplus b \in E_{S}$.

Obviously, $E_{S}$ is an orthoalgebra.

Since, for a homogeneous effect algebra $E, E_{S}$ is an orthoalgebra, every compatible subset of $E_{S}$ can be embedded into a block of $E_{S}$, which is a Boolean algebra.

Proposition 4.5. Let $E$ be a homogeneous effect algebra. For every block $B^{0}$ in $E_{S}$ and for every block $B$ of $E$ such that $B^{0} \subseteq B, B^{0}=C(B)$.

Proof: Let $B^{0}$ be a block of $E_{S}$. Let $B$ be a block of $E$ with $B^{0} \subseteq B$. By Proposition $4.2, B^{0} \subseteq C(B)$. Since $B^{0}$ is a block of $E_{S}$ and $C(B)$ is a Boolean algebra, $B^{0} \subseteq C(B)$ implies that $B^{0}=C(B)$.

QUESTION 4.6. Let $B$ be a block of a homogeneous effect algebra $E$. Is it true that $C(B)$ is a block of $E_{S}$ ?

Proposition 4.7. In a homogeneous effect algebra, $C(E)=C(K(E))=$ $K(E)_{S}$.

Proof: It is evident that $C(E) \subseteq C(K(E)) \subseteq K(E)_{S}$. Let $a \in K(E)_{S}$. We shall prove that $[0, a]$ is a Riesz ideal. By Lemma 2 of [5], this implies that $a \in C(E)$. Suppose $x_{1}, x_{2} \leqslant a, x_{1} \perp x_{2}$. Then $\left\{x_{1}, x_{2}\right\}$ can be embedded into a block $B$ of $E$. Since $a \in K(E), a \in B$. Since $a$ is sharp; $a$ is central in $B$. Thus, $a$ is principal in $B$ and hence $x_{1} \oplus x_{2} \leqslant a$. Therefore, $a$ is principal in $E$. Let $i \in[0, a], x \perp y, i \leqslant x \oplus y$. Similarly as above, $\{a, x, y\}$ can be embedded into a block $B$ of $E$, such that $a \in C(B)$. Obviously, $i \leqslant(x \oplus y) \wedge a$ and, since $a$ is central in $B,(x \oplus y) \wedge a=(x \wedge a) \oplus(y \wedge a)$. Thus, $[0, a]$ is a Riesz ideal. 


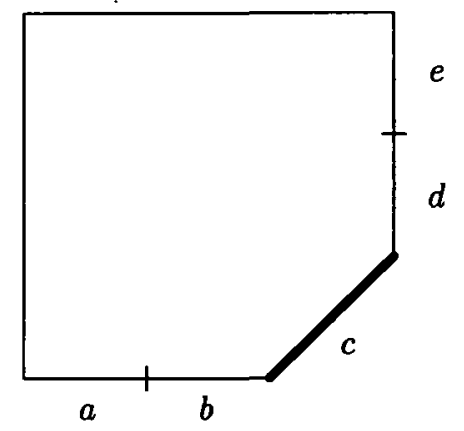

Figure 1.

QUESTION 4.8. Let $E$ be a homogeneous effect algebra. Does $K(E)$ satisfy the Riesz decomposition property? This is true for orthoalgebras and for lattice ordered effect algebras.

\section{EXAMPLES AND COUNTEREXAMPLES}

It is easy to check that a direct product of a finite number of homogeneous effect algebras is a homogeneous effect algebra.

EXAMPLE 5.1. Let $E_{1}$ be an orthoalgebra. Let $E_{2}$ be an effect algebra satisfying the Riesz decomposition property, which is not an orthoalgebra. If either of $E_{1}, E_{2}$ is not lattice ordered, then $E_{1} \times E_{2}$ is an example of a homogeneous effect algebra which is not lattice ordered. Moreover, since $E_{2}$ is not an orthoalgebra, $E_{1} \times E_{2}$ is not an orthoalgebra.

Another possibility to construct new homogeneous effect algebras from old is to make horizontal sums (sometimes called 0,1-pastings), which means simply identifying the zeros and ones of the summands.

As shown in the next example, it is possible to construct a lattice ordered (and hence homogeneous) effect algebra by pasting of two MV-algebras in a central element.

Example 5.2. We borrowed the basic idea for this example from Cohen [7]. Consider a system consisting of a firefly in a box pictured in a Figure 1. The box has five windows, separated by thin lines. We shall consider two experiments on this system :

(A) Look at the windows $a, b, c$.

(B) Look at the windows $c, d, e$.

Suppose that the window $c$ is covered with a grey filter. Unless the firefly is shining very brightly at the moment we are performing the experiment, we cannot be sure that we see the firefly in the $c$ window. The outcomes of experiment (A) are 


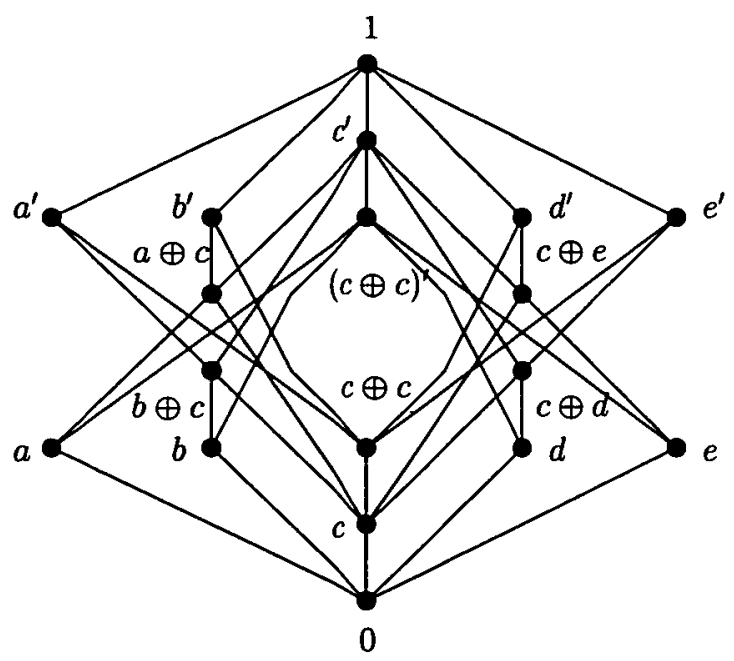

Figure 2: An eighteen element lattice ordered effect algebra

(a) We see the firefly in window $a$.

(b) We see the firefly in window $b$.

(c) We see the firefly in window $c$, with the level of (un)certainity $1 / 2$.

$(c \oplus c)$ We see the firefly in window $c$.

The outcomes of (B) are similar. The unsharp quantum logic of our experiment is an eighteen element lattice ordered effect algebra $E$ with five atoms $a, b, c, d, e$, satisfying

$$
a \oplus b \oplus c \oplus c=c \oplus c \oplus d \oplus e=1
$$

The Hasse diagram of $E$ is given by Figure 2. This effect algebra is constructed by pasting two MV-algebras

$$
A=\left\{0, a, b, c, a \oplus c, b \oplus c, c \oplus c, a^{\prime}, b^{\prime},(c \oplus c)^{\prime}, c^{\prime}, 1\right\}
$$

and

$$
B=\left\{0, c, d, e, c \oplus c, c \oplus d, c \oplus e, d^{\prime}, e^{\prime},(c \oplus c)^{\prime}, c^{\prime}, 1\right\}
$$

$A$ and $B$ are then blocks of $E$. The compatibility centre of $E$ is the MV-algebra

$$
K(E)=\left\{0, c, c \oplus c,(c \oplus c)^{\prime}, c^{\prime}, 1\right\}
$$

and the centre of $E$ is $\left\{0, c \oplus c,(c \oplus c)^{\prime}, 1\right\} . E_{S}$ forms a twelve-element orthomodular lattice with two blocks; each of them is isomorphic to the Boolean algebra $2^{3}$ and they are pasted in one of their atoms (namely $c \oplus c$ ). 


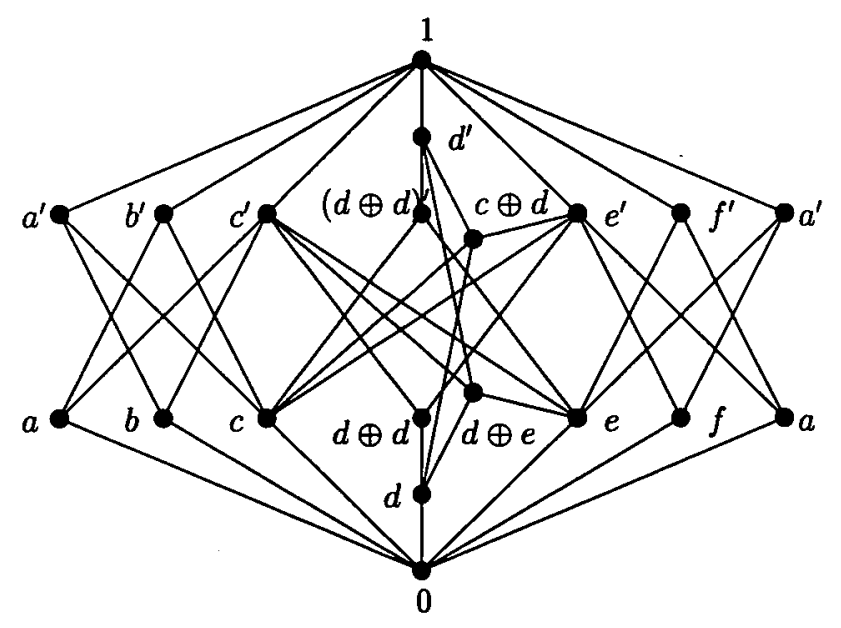

Figure 3: A non-lattice ordered homogeneous effect algebra

EXAMPLE 5.3. Let $E$ be an eighteen element effect algebra with six atoms $a, b, c, d, e, f$, satisfying

$$
a \oplus b \oplus c=c \oplus d \oplus d \oplus e=e \oplus f \oplus a=1
$$

The Hasse diagram of $E$ is given by Figure 3 .

This effect algebra is constructed by pasting of three blocks: two Boolean algebras

$$
\begin{aligned}
& B_{1}=\left\{0, a, b, c, a^{\prime}, b^{\prime}, c^{\prime}, 1\right\} \\
& B_{2}=\left\{0, e, f, a, e^{\prime}, f^{\prime}, a^{\prime}, 1\right\}
\end{aligned}
$$

and an MV-algebra

$$
B_{3}=\left\{0, c, d, e, d \oplus d, d \oplus e, c \oplus d,(d \oplus d)^{\prime}, c^{\prime}, d^{\prime}, e^{\prime}, 1\right\} .
$$

By (3), it is easy to see that the range of every orthogonal family with three elements can be embedded into a block. Thus, by Corollary 3.14, $E$ is homogeneous. All elements except for $d, d^{\prime}, c \oplus d, d \oplus e$ are sharp and $E_{S}$ is an orthoalgebra with fourteen elements, called the Wright triangle, which is not an orthomodular poset.

Propos I TION 5.4. Let $E$ be a homogeneous effect algebra. Assume that there is an element $a \in E$ with $a \leqslant a^{\prime}$, such that $E$ is isomorphic to $[0, a]_{E}$. Then $E$ satisfies the Riesz decomposition property.

Proof: Let $B$ be a block containing $a$. Since $B$ is a maximal internally compatible subset of $E$, Corollary 3.8(b) implies that $[0, a]=\left\{x \in E: x \leqslant a, a^{\prime}\right\} \subseteq B$. This implies 
that $[0, a]_{E}$ satisfies the Riesz decomposition property. Therefore, $E$ satisfies the Riesz decomposition property.

Corollary 5.5. For a Hilbert space $\mathbb{H}, \mathcal{E}(\mathbb{H})$ is homogeneous if and only if $\operatorname{dim}(\mathbb{H}) \leqslant 1$.

Proof: The map $\phi: \mathcal{E}(\mathbb{H}) \mapsto[0, I / 2]$ given by $\phi(A)=A / 2$ is obviously an isomorphism and $I / 2 \leqslant(I / 2)^{\prime}$. Therefore, by Proposition 5.4, every homogeneous $\mathcal{E}(\mathbb{H})$ satisfies the Riesz decomposition property. However, it is well known that $\mathcal{E}(\mathbb{H})$ satisfies the Riesz decomposition property if and only if $\operatorname{dim}(\mathbb{H}) \leqslant 1$.

The following example shows that a sub-effect algebra of a homogeneous effect algebra need not to be homogeneous.

EXAMPLE 5.6. Let $E=[0,1] \times[0,1]$, where $[0,1] \subseteq \mathbb{R}$ denotes the unit interval of the real line. Equip $E$ with a partial operation $\oplus$ with domain given by $\left(a_{1}, a_{2}\right) \perp\left(b_{1}, b_{2}\right)$ if and only if $a_{1}+b_{1} \leqslant 1$ and $a_{2}+b_{2} \leqslant 1$; then define $\left(a_{1}, a_{2}\right) \oplus\left(b_{1}, b_{2}\right)=\left(a_{1}+b_{1}, a_{2}+b_{2}\right)$. Then $\left(E, \oplus_{E},(0,0),(1,1)\right)$ is a homogeneous effect algebra (in fact, it is even an MValgebra). Let

$$
F=\left\{\left(x_{1}, x_{2}\right) \in E: x_{1}+x_{2} \in \mathbb{Q}\right\} .
$$

Since $(1,1) \in F$ and $F$ is closed with respect to $\Theta, F$ is a sub-effect algebra of $E$.

It is easy to see that the map $\phi: F \mapsto[(0,0),(1 / 2,1 / 2)]_{F}$, given by $\phi\left(x_{1}, x_{2}\right)=$ $\left(x_{1} / 2, x_{2} / 2\right)$ is an isomorphism. Note that $F$ is not a compatible effect algebra: for example, $\{(1,0),(1 / \pi, 1-(1 / \pi))\}$ is not compatible in $F$. Consequently, $F$ does not satisfy the Riesz decomposition property and hence, by Proposition 5.4, $F$ is not homogeneous.

EXAMPLE 5.7. Let $\mu$ be the Lebesgue measure on $[0,1]$. Let $E \subseteq[0,1]^{[0,1]}$ be such that, for all $f \in E$,

(a) $f$ is measurable with respect to $\mu$

(b) $\mu(\operatorname{supp}(f)) \in \mathbb{Q}$

(c) $\mu(\{x \in[0,1]: f(x) \notin\{0,1\}\})=0$,

where $\operatorname{supp}(f)$ denotes the support of $f$. It is easy to check that $E$ is a sub-effect algebra of $[0,1]^{[0,1]}$. Obviously, $E$ is not an orthoalgebra. We shall show that $E$ is a homogeneous, non-lattice ordered effect algebra and that $E$ does not satisfy the Riesz decomposition property. Note that, for all $u \in E, u \perp u$ if and only if $\operatorname{Ran}(u) \subseteq[0,1 / 2]$ and $\mu(\operatorname{supp}(u))=0$. Thus, for all $u \in E$ and $u_{0} \in[0,1]^{[0,1]}$ such that $u_{0} \leqslant u$ and $u \perp u$, we have $u_{0} \in E$.

Let $u, v_{1}, v_{2} \in E$ be such that $u \leqslant v_{1} \oplus v_{2}, u \leqslant\left(v_{1} \oplus v_{2}\right)^{\prime}$. Since $[0,1]^{[0,1]}$ is an MV-algebra, there are $u_{1}, u_{2} \in[0,1]^{X}$ such that $u_{1} \leqslant v_{1}, u_{2} \leqslant v_{2}$ and $u=u_{1} \oplus u_{2}$. By the above paragraph, $u \perp u$ and $u_{1}, u_{2} \leqslant u \in E$ imply that $u_{1}, u_{2} \in E$. Therefore, $E$ is homogeneous. Let $f, g$ be the characteristic functions of the intervals $[0,2 / 3]$, $[1 / \pi,(1 / \pi)+(1 / 2)]$, respectively. Then $f \wedge g$ does not exist in $E_{S}$. Therefore, $E_{S}$ is not 
lattice ordered and hence, by Theorem 3.3 of [13], $E$ is not lattice ordered. Moreover, $E$ does not satisfy the Riesz decomposition property. Indeed, assume the contrary. Then, by Proposition 4.3, $E_{S}=C(E)$. In particular, $E_{S}$ is then a Boolean algebra. However, this is a contradiction, since $E_{S}$ is not lattice ordered.

\section{REFERENCES}

[1] M.K. Bennett and D.J. Foulis, 'Phi-symmetric effect algebras', Foundations of Physics 25 (1995), 1699-1722.

[2] L. Beran, Orthomodular lattices. Algebraic approach. (D. Reidel Publishing Co., Dordrecht, 1985).

[3] P. Bush, M. Grabowski, and P. Lahti, Operational quantum physics (Springer-Verlag, Berlin, Heidelberg, New York, 1995).

[4] C.C. Chang, 'Algebraic analysis of many-valued logics', Trans. Amer. Math. Soc. 89 (1959), 74-80.

[5] G. Chevalier and S. Pulmannová, 'Some ideal lattices in partial abelian monoids', (preprint, Mathematical Institute of Slovak Academy of Sciences, Bratislava, 1998).

[6] F. Chovanec and F. Kôpka, 'Boolean D-posets. Quantum structures', Tatra Mt. Math. Publ. 10 (1997), 1-15.

[7] D.W. Cohen, An introduction to Hilbert space and quantum logic (Springer-Verlag, Berlin, Heidelberg, New York, 1989).

[8] D.J. Foulis and M.K. Bennett, 'Effect algebras and unsharp quantum logics', Found. Phys. 24 (1994), 1331-1352.

[9] D.J. Foulis, R. Greechie and G. Rütimann, 'Filters and supports in othoalgebras', Internat. J. Theoret. Phys. 35 (1995), 789-802.

[10] R. Giuntini and H. Greuling, 'Toward a formal language for unsharp properties', Found. Phys. 19 (1994), 769-780.

[11] R. Greechie, D. Foulis and S. Pulmannová, 'The center of an effect algebra', Order 12 (1995), 91-106.

[12] S. Gudder and S. Pulmannová, 'Quotients of partial abelian monoids', Algebra Universalis 38 (1998), 395-421.

[13] G. Jenča and Z. Riečanová, 'On sharp elements in lattice ordered effect algebras', BUSEFAL 80 (1999), 24-29.

[14] G. Kalmbach, Orthomodular lattices (Academic Press, New York, 1983).

[15] F. Kôpka and F. Chovanec, 'D-posets', Math. Slovaca 44 (1994), 21-34.

[16] G. Ludwig, Foundations of quantum mechanics (Springer-Verlag, Berlin, Heidelberg, New York, 1983).

[17] P. Pták and S. Pulmannová, Orthomodular structures as quantum logics (Kluwer, Dordrecht, 1991).

[18] K. Ravindran, On a structure theory of effect algebras, (Ph.D. thesis) (Kansas State Univ., Manhattan, KS, 1996).

[19] Z. Riečanová, 'Compatibility and central elements in effect algebras', Tatra Mt. Math. Publ. 16 (1998), 1-8. 
[20] Z. Riečanová, 'A generalization of blocks for lattice effect algebras', Int. J. Theor. Phys. $39(2000), 855-865$.

Department of Mathematics

Faculty of Electrical Engineering and Information Technology

Ilkovičova 3

81219 Bratislava

Slovakia

e-mail: jenca@kmat.elf.stuba.sk 